\title{
Does Neutrophil Lymphocyte Ratio Have a Clinical Value to Determine the Severity of the Patients with Acute Appendicitis?
}

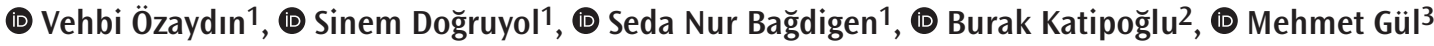 \\ 1Department of Emergency Medicine, Medeniyet University Göztepe Training and Research Hospital, İstanbul, Turkey \\ 2Department of Emergency Medicine, Ankara Training and Research Hospital, Ankara, Turkey \\ ${ }^{3}$ Department of Emergency Medicine, Konya Necmettin Erbakan University Meram Faculty of Medicine, Konya, Turkey
}

\begin{abstract}
Aim: The aim of our study is to investigate the predictive value of the neutrophil lymphocyte ratio (NLR) assessed in the emergency department to distinguish complicated and uncomplicated patients with acute appendicitis (AA).

Materials and Methods: In our study, the files of the patients with AA who visited our emergency clinic between 01.06.2015 and 01.01.2016 and then were operated in our hospital were reviewed retrospectively. The age, gender, the imaging method performed in the emergency clinic with an AA preliminary diagnosis, the result of the radiologic report in terms of AA and histopathological examination results were recorded according to the patients' records. The patients were divided into two groups: Complicated AA (group 1) and uncomplicated AA (group 2) based on their histopathological analysis results. The laboratory parameters of the patients, which were seen within the first 30 minutes after they visited the emergency clinic, were examined.

Results: One hundred and twenty one of the 154 patients who were involved in the study were in group 1 and 33 of the patients were in group 2. A statistically significant difference was found between two groups in terms of the number of white blood cells and NLR ( $p=0.000$ ). The cut-off value for NLR in the complicated AA distinction was detected as 7.3 (75.8\% sensitivity, 81.8\% specifity).

Conclusion: As an easy and effective analysis method, we think that NLR might be a good guide to diagnose complicated patients with AA quickly.

Keywords: Acute abdomen, complicated appendicitis, laboratory parameters
\end{abstract}

\section{Introduction}

Acute appendicitis (AA) is one of the most common causes of acute abdomen in emergency services. Although the patients usually appear to have characteristic symptoms, the number of patients with atypical complaints is not low to be underestimated (1). Despite the widespread use of imaging modalities such as ultrasound (US) and computed tomography (CT), the clinical diagnosis of AA is still challenging (2). In this process, systems that use more than one step give more satisfactory results than a single diagnostic method. These diagnostic approaches may dramatically reduce negative appendectomies, perforation numbers, and the time spent in the hospital (3).
In many parts of our country, there are centers that cannot benefit from imaging methods. In these centers, the most important diagnostic tool for AA is a careful anamnesis and a good physical examination supported by simple laboratory results. There are many studies evaluating the number of leukocytes in whole blood count in the case of AA. Differently, the neutrophil lymphocyte ratio (NLR) is a relatively new marker for studies and is associated with poor survival in many diseases $(4,5)$. For this purpose, studies have been carried out to test if NLR can distinguish complicated and uncomplicated patients with AA $(6,7)$.

The aim of our study is to investigate the predictive value of the NLR assessed in the emergency department to distinguish complicated and uncomplicated patients with AA. All of the patients in this 
study were examined with AA prediagnosis and operated after evaluating with at least one of the imaging modalities to confirm the AA diagnosis. The postoperative pathological results of the patients were evaluated retrospectively to determine whether they were complicated.

\section{Materials and Methods}

The patients with AA who visited the emergency clinic in a thirdstage emergency department of a university hospital and were operated in the hospital during a 6-month period from June 1, 2015, to January 1, 2016, were reviewed retrospectively. The ethics committee's approval for the study was given by the same institution.

The files of the patients between the ages of 18-65 years who were operated by our hospital's general surgery department after visiting the emergency clinic and had complaints compatible with AA and underwent at least one imaging modality (US and/ or (T) in our hospital's records during diagnostic process in the emergency clinic were reviewed. The official radiology reports of the imaging method in all these patients were available in our hospital system. The age, gender, the imaging method performed in the emergency clinic with an AA preliminary diagnosis, the result of the radiologic report in terms of $A A$ and histopathological examination results were recorded according to the patients' records. The patients were divided into two groups: Complicated AA (group 1) and uncomplicated AA (group 2) based on their histopathological analysis results. As a result of histopathology results, the ones that had gangrenous appendicitis, perforated appendicitis, plastron appendicitis, periapical abscesses were considered as complicated AA. The white blood cell (WBC), neutrophil, and lymphocyte counts were determined by analyzing the laboratory parameters taken from the peripheral vena in the first $30 \mathrm{~min}$ of patients' visit to the emergency department. The NLR value was calculated by dividing the number of neutrophils by the number of lymphocytes. The reference ranges were $4000-10000 / \mathrm{mm}^{3}$ for WBC and $1400-6500 /$ $\mathrm{mm}^{3}$ for lymphocytes in terms of laboratory parameters. The NLR ratios of both group 1 and group 2 were compared individually. The patients who had acute and chronic diseases which could affect laboratory parameters through inflammation markers were not included in this study.

\section{Statistical Analysis}

For statistical evaluation of the data, the statistical package software of IBM Statistics 20.0 (SPSS) was used. The suitability of continuous variables to normal distribution was assessed using the Kolmogorov-Smirnov test. The Student's t-test was used for the binary group comparisons of normally distributed data. The chi-square test was used to compare categorical variables.
Percentage, frequency, mean, and standard deviation were given as descriptive statistics. The receiver operating characteristic (ROC) analysis was used to determine the diagnostic and cutoff values of NLR in complicated patients with AA. In the obtained ROC curve, the proximity of the area under the curve (AUC) value of 1 indicated a high value of the significance level of the test. The level of significance for the AUC obtained in the same test was also determined. The results were evaluated in a 95\% confidence interval $(\mathrm{Cl})$ and at a significance level of $p<0.05$.

\section{Results}

Of the 154 patients studied, 93 (60.4\%) were male and 61 (39.6\%) were female. The mean age of the males was $36.39 \pm 12.69$ years and the mean age of the females was $33.29 \pm 10.38$. There was no significant difference in age distribution among the sexes $(\mathrm{p}>0.005)$.

There were 121 patients in group 1 and 33 patients in group 2. There was no significant difference between the two groups in terms of gender $(p>0.005)$. The mean age of all patients was revealed as $34.52 \pm 11.41$ years and when the mean age of the patients was examined, there was no significant difference between group 1 and group 2 ( $p>0.005)$. However, a significant difference was noted in the WBC count between the two groups (mean difference, 2.9; 95\% $\mathrm{Cl}=1.316-4.508)(\mathrm{p}=0.000$ ). A significant difference was found between the two groups in terms of NLR. The NLR values of the patients in group 2 were found to be higher compared with the values of the patients in group 2 (mean difference, 6.4; 95\% Cl=4.952-7.807) $(\mathrm{p}=0.000)$ (Table 1).

When the imaging method in the diagnosis process was evaluated, it was seen that 126 out of 154 cases were diagnosed with CT, 73 with CT and 45 with both US and CT. One hundred and nine cases (70.8\%) were diagnosed with a single imaging method while both imaging methods were used in 45 cases (29.2\%). There was no significant difference between groups in terms of selection of individual imaging methods ( $p>0.005)$. There was no significant difference between the groups in terms of the number of patients using both imaging methods ( $p>0.005$ ).

\begin{tabular}{|c|c|c|c|c|}
\hline \multicolumn{2}{|c|}{ Variant group } & $\begin{array}{l}\text { Group } 1 \\
(\mathrm{n}=121)\end{array}$ & $\begin{array}{l}\text { Group } 2 \\
(\mathrm{n}=33)\end{array}$ & p \\
\hline \multirow{2}{*}{ Gender } & Male & $76(62.8 \%)$ & $17(51.5 \%)$ & $>0.005$ \\
\hline & Female & $45(37.2 \%)$ & $16(48.5 \%)$ & - \\
\hline \multicolumn{2}{|l|}{ Age } & $34 \pm 10.5$ & $37 \pm 14.2$ & $>0.005$ \\
\hline \multicolumn{2}{|c|}{ Leukocyte } & $13302 \pm 3686$ & $16215 \pm 5417$ & 0.000 \\
\hline \multicolumn{2}{|c|}{ Neutrophil lymphocyte ratio } & $5.2 \pm 3.1$ & $11.6 \pm 5.6$ & 0.000 \\
\hline
\end{tabular}


The NLR thresholds were calculated with ROC curve analysis. The AUC for NLR was calculated as 0.856 (standard error, 0.038; $95 \% \mathrm{Cl}=0.781-0.930)(p=0.000)$. For $\mathrm{NLR}$, the cut-off value was found to be 7.3 (75.8\% sensitivity, $81.8 \%$ selectivity) and 3.3 was determined as a threshold value which could be used for exclusion and 16.8 could be used as threshold value for diagnosis (Figure 1).

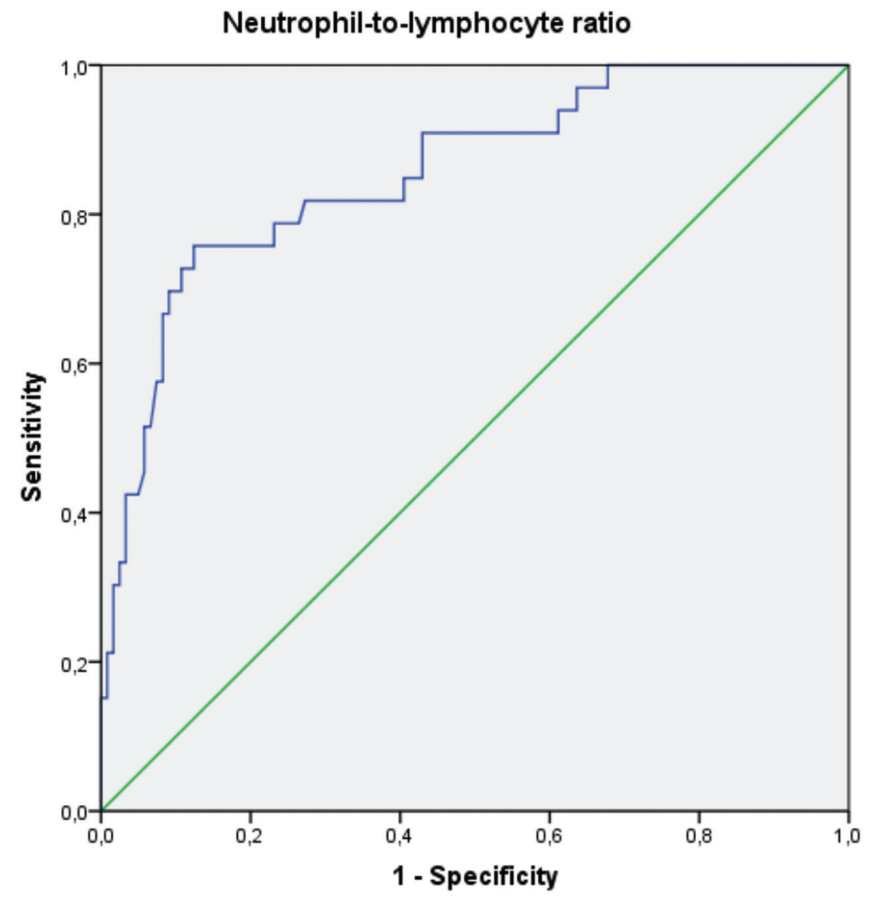

Figure 1. Receiver-operating characteristic curve for neutrophilto-lymphocyte ratios in complicated appendicitis Area under the curve was 0.856 (standard error, 0.038; 95\% Cl=0.781-0.930). Ideal cutoff value was 7.3 , this cutoff value yielded sensitivity of $75.8 \%$ and specificity of $81.8 \%$

$\mathrm{Cl}$ : Confidence interval

\section{Discussion}

The early diagnosis of AA is still a challenge. The perforation incidence in patients with AA is between $18.3 \%$ and $34 \%$ (8). As only the perforation incidence is considered to be quite high, the need for simple and useful tests becomes important for the early recognition of complicated patients.

Previous studies have shown that high WBC in total blood count is sensitive to indicate appendix inflammation (9). However, as the high WBC does not seem to be specific in AA, different tests should be considered as well. The most valuable diagnostic tool is the total blood count following anamnesis and physical examination in the rural areas where imaging methods cannot be used in the diagnosis process. Therefore, the parameter that will lead to the early diagnosis of patients with complicated AA should be selected from basic blood parameters. In the light of the studies analyzing basic blood parameters, the individual value of NLR in the evaluation of AA complications has recently become quite popular (4).

In a similar study, the cut-off value of NLR was 7.95, and it was reported to have high sensitivity and specificity to distinguish complicated patients with AA from uncomplicated patients (10). It was thought that the increase in NLR seen in complicated patients with AA occurred due to a decrease in the number of lymphocytes in severe patients which was reported in the literature a long time ago (11). In this study, a similar cut-off value was found $(3,7)$ in terms of differentiating complicated patients with AA. Unlike in the literature, the specificity ratio at the cut-off value was higher in this study.

The WBC counts in the complicated patients with AA were significantly higher compared with the WBC count in the other patient groups. In accordance with the literature, WBC values were found to be significantly higher in complicated patients with AA (12). Thus, WBC count can be used for the early diagnosis of complicated patients with $\mathrm{AA}$ and is correlated with histopathological results. Although the increase in WBC count was used as a parameter in the AA diagnostic process earlier, it still remained effective to assess the severity of the cases.

From the demographic point of view, it was determined that there was a significant difference between the uncomplicated and complicated patients with AA according to gender in our country. The frequency of complicated AA was higher in women and uncomplicated AA frequency was higher in men (7). In our study, we did not find any significant difference between the patient groups in terms of gender. Similarly, no significant difference was found in terms of age distribution among the groups. As far as we can tell from the literature, there is no study evaluating the use of NLR to guide the choice of imaging method in AA. Kim et al. (13) evaluated the correlation of CT outcomes with laboratory test outcomes and explained the contribution of both tests. In our study, we evaluated the imaging modalities performed during the diagnostic process of patients with AA and did not find any significant difference between the patient groups that we categorized according to the complications. However, since this was a retrospective study, a prospective study might be useful in evaluating the guiding power of NLR to decide which imaging method would be better in the diagnostic process.

\section{Study Limitations}

The limitation of this study was that it was designed retrospectively and performed by examining the data in the patient files. A prospectively designed study contributing to scoring systems in 
AA can be conducted to determine the severity of patients with AA using NLR. The NLR cutoff value determined in this study might serve as a guide due to its high specificity and sensitivity rates.

\section{Conclusion}

The early recognition of complicated patients with AA is a primary task of emergency medicine specialists and general surgery specialists. AA accounts for a large proportion of the patients diagnosed in terms of acute abdomen in emergency departments. NLR can be used as an easy and useful diagnostic tool in the rapid recognition of complicated patients with AA in rural areas where it is difficult to access imaging methods.

\section{Ethics}

Ethics Committee Approval: Retrospective study..

Informed Consent: Retrospective study.

Peer-review: Externally peer-reviewed.

\section{Authorship Contributions}

Concept: V.Ö., S.D., Design: S.D., B.K., Data Collection or Processing: S.N.B., V.Ö., Analysis or Interpretation: M.G., B.K., Literature Search: S.N.B, Writing: S.D., V.Ö.

Conflict of Interest: No conflict of interest was declared by the authors.

Financial Disclosure: The authors declared that this study received no financial support.

\section{References}

1. Cardall T, Glasser J, Guss DA. Clinical value of the total white blood cell count and temperature in the evaluation of patients with suspected appendicitis. Acad Emerg Med. 2004;11:1021-27.
2. Flum DR, McClure TD, Morris A, Koepsell T. Misdiagnosis of appendicitis and the use of diagnostic imaging. J Am Coll Surg. 2005;201:933-9.

3. Denizbasi A, Unluer EE. The role of the emergency medicine resident using the Alvarado score in the diagnosis of acute appendicitis compared with the general surgery resident. Eur J Emerg Med. 2003;10:296-301.

4. Markar SR, Karthikesalingman A, Falzon A, Kan Y. The diagnostic value of neutrophil: lymphocyte ratio in adults with suspected acute appendicitis. Acta Chir Belg. 2010;110:543-7.

5. Yazici M, Ozkisacik S, Oztan OM, Gursoy H. Neutrophil/lymphocyte ratio in the diagnosis of childhood appendicitis. Turk J Pediatr. 2010;52:400-3.

6. Yazar FM, Bakacak M, Emre A, Urfalioglu A, Serin S, Cengiz E, et al. Predictive role of neutrophil-to-lymphocyte and platelet-to-lymphocyte ratios for diagnosis of acute appendicitis during pregnancy. Kaohsiung J Med Sci. 2015;31:591-6

7. Yildirim AC, Anuk T, Gunal E, Irem B, Gulkan S, Dayilar H, et al. Clinical value of the platelet-to-lymphocyte ratio for diagnosing complicated acute appendicitis. Turk J Colorectal Dis 2017;27:1-5

8. McGowan DR, Sims HM, Zia K, Uheba M, Shaikh IA. The value of biochemical markers in predicting a perforation in acute appendicitis. ANZ J Surg. 2013;83:79-83.

9. Sevinc MM, Kınaci E, Çakar E, Bayrak S, Özakay A, Aren A, et al. Diagnostic value of basic laboratory parameters for simple and perforated acute appendicitis: an analysis of 3392 cases. Ulus Travma Acil Cerrahi Derg. 2016;22:155-62.

10. Yardimci S, Ugurlu MÜ, Coskun M, Attaallah W, Yegen SC. Neutrophillymphocyte ratio and mean platelet volume can be a predictor for severity of acute appendicitis. Ulus Travma Acil Cerrahi Derg. 2016;22:163-8.

11. Hoffmann J, Rasmussen 00. Aids in the diagnosis of acute appendicitis. $\mathrm{Br} J$ Surg. 1989;76:774-9.

12. Yang HR, Wang YC, Chung PK, Chen WK, Jeng LB, Chen RJ. Laboratory tests in patients with acute appendicitis. ANZ J Surg. 2006;76:71-4.

13. Kim HC, Yang DM, Lee CM, Jin W, Nam DH, Song JY, et al. Acute appendicitis: relationships between $\mathrm{CT}$-determined severities and serum white blood cell counts and C-reactive protein levels. Br J Radiol. 2011;84:1115-20. 